small apertures above and below it about $1 \frac{1}{2}$ millimetres in diameter This lamina is probably the result of ossification of granulations due to an external otitis. There are no apparent detp-seated changes.

The other specimen, a left temporal bone, was removed from a female, aged eighty, whose meatus was seen during life to be oceluded by an epithelial covered membrane. The ear was totally deaf, anil from examination of the specimen the occlusion is seen to be due to an atrophic epidermal layer lying on, and continuous with, some fatty commective tissue which occupied the site of the midllle ear and could be seen passing deeply into the situation of the vestibule, which was disorganised. No trace remained of the semicircular canals and the mastoid cells were full of solid connective tissue. The cochlea was much disorganised, the spiral ganglion being the only remnant of normal structure, the basal coil being filled with new bone and the remainder with fibrous tissue. The bonv new formation seems to have arisen from the endosteum of the labyrintil. The specimen probably demonstrates the spontaneous recovery of a suppurative labyriuthitis.

A. J. Wright.

\title{
PHARYNX AND CESOPHAGUS.
}

\section{Gastinel. P., and Pelissier, Andre (Paris),-Syphilis, Diphtheritic Paralysis, Palatine Herpes. " Gaz. des Hop.," Octoler 5, 191 !.}

The authors remark that ulcero-vesicular eruptions are recognised facts and generally involve no difficulty in diagnosis. There are, however, cases where such conditions occur in subjects of infection, apart from neuritis, and then it is a delicate matter to discriminate between trophic phenomena and what may be due to infection. The following case affords an example. In August, 1910, a woman had a vulvar chancre which had never been treated. In October of the same year she had diphtheria and was treated with Roux's serum. Paralysis of the soft palate ensued and lasted three weeks. At the end of November small vesicles appeared on the velum, which ulcerated and shortly cicatrised, but reappeared some days afterwards. Similar recurrences took place six times in two months. The patient's attention was only drawn to them by slight dysphagia. When seen in January there was a diffuse redness of the entire pharynx and velum. On the latter, and quite limited to its posterior part, a crop of vesicles was observed, some pearly, others had hurst. They extended on to the anterior pillar of the fances, but respected the tonsils, pesterior pharyngeal wall, cheeks and tongue. The eruption was unaccompanied l,y fever, headache or functional srmptoms, except slight dysphagia. 'There was no spontaneous pain. Paralysis of the palate had disappeared and its sensibility was intact. At this time the patient manifested secondary syphilitio symptoms. The trunk and limbs were covered with a maculo-papular rash, and hypertrophic mucous plaques were present on the vulva. There were no inucous patches on the lips, tougue or pharyn. Some days afterwards the vesicles had completely disappeared, giving place to polycyclic erosions. The pharynx was red, but no lesions were present. The syphilides had the same distribution and always respected the bucco-pharyngeal cavity. The nature of this herpetiform eruption occurring in a syphilitic subject, on a territory previously attacked by neuritis, and also the relation which the specific infection and nerve lesion may have had to the eruption, are fully discussed.

H. Clayton Fox. 